This is the peer reviewed version of the following article: Ashok PC, Giardini ME, Dholakia K, Sibbett W (2014) A Raman spectroscopy bio-sensor for tissue discrimination in surgical robotics, Journal of Biophotonics 7, No. 1-2, 103-109

DOI :10.1002/jbio.201300034, which has been published in final form at

http://onlinelibrary.wiley.com/doi/10.1002/jbio.201300034/abstract. This article may be used for

non-commercial purposes in accordance with Wiley Terms and Conditions for Self-Archiving

\title{
Raman spectroscopy bio-sensor for tissue discrimination in surgical robotics
}

\author{
Praveen C. Ashok ${ }^{1}$, Nikola Krstajic ${ }^{1}$, Mario E. Giardini ${ }^{2}$, Kishan Dholakia ${ }^{1}$, Wilson Sibbett ${ }^{1}$ \\ ${ }^{1}$ SUPA School of Physics and Astronomy, University of St Andrews, North Haugh, St Andrews KY16 \\ 9SS, UK \\ ${ }^{2}$ School of Medicine, Medical and Biological Sciences Building, University of St Andrews, North \\ Haugh, St Andrews, KY16 9TF, UK
}

Keywords: Raman spectroscopy, Fiber probe, Tissue diagnostics, Surgical robotics, Medical optics

We report the development of a fiber based Raman sensor to be used in endoluminal robotic surgery. Although this is a generic platform, the sensor mentioned in this article was adapted for the ARAKNES (Array of Robots Augmenting the KiNematics of Endoluminal Surgery) robotic platform. On such platform, the Raman sensor is intended to identify ambiguous tissue margins during robot-assisted surgeries. To maintain sterility of the probe during surgical intervention, a disposable sleeve was specially designed. A straightforward user-compatible interface was implemented where a supervised multivariate classification algorithm was used to classify different tissue types based on its Raman fingerprints so that it could be used without any knowledge of spectroscopic data analysis. The protocol avoids inter-patient variability in data and the system itself is not limited to be used in the classification of a specific tissue type. We performed ex vivo tissue classification assessments with this system where it showed $>95 \%$ sensitivity and specificity for tissue classification. 


\section{Introduction}

Access to complementary multimodal information regarding the tissue morphology and chemical composition can be of significant assistance to surgeons during robot- assisted or laparoscopic surgery. Various optical technologies can be used to obtain this type of information that can inform the surgical decisions regarding the incision margin during relevant procedures. Raman spectroscopy is a technique that can provide bio-chemical information of the sample which can then be used to discriminate between different tissue types [1]. In this respect, the development of fiber Raman probes has enabled in vivo tissue studies using Raman spectroscopy [2-4]. A Raman sensor that can act as an accessory to a surgical robot in turn represents a significant device for tissue margin identification. An attempt towards developing a prototype bio-sensor based on Raman spectroscopy to assist image-guided surgery has already been reported [5]. However this study only focused at the algorithm to use Raman spectroscopic sensing in surgical robotics. The study used a bulk Raman probe (probe head diameter $\sim 1 \mathrm{~cm}$, length of the rigid part of probe head $\sim 0.5 \mathrm{~m}$ ) which did not allow easy maneuvering during a real surgical environment. It also did not address the issues related to development of surgery-compatible hardware with respect to geometrical constraints and to sterilizability, or classification software signficant in a practical implementation in a real surgical environment.

Here we report on the implementation of a full Raman-based sensing system (instrumentation, protocol and software) that can provide real-time analytical information and guidance during the surgical procedures. The device in this instance is designed with regard to the second-generation surgical robot platform - ARAKNES (Array of Robots Augmenting the KiNematics of Endoluminal Surgery) but the principles may be adapted to other robotic platforms [6]. A particular facet of the device was that the hardware was designed to be mechanically compatible with the ARAKNES robot. A sterile disposable sleeve ensures sterility of the fiber probe during surgical intervention. A simple protocol based upon supervised multivariate algorithm was developed for binary tissue discrimination and the software interface for this simple protocol can be operated by a person who does not have any prior experience in spectroscopy. The ability of this sensor to discriminate different tissue types has been demonstrated at a proof-of-principle level through ex-vivo tissue discrimination studies.

\section{Instrumentation}

The optical hardware of the Raman sensor consists of three principal components - a laser, a spectrometer and a fiber Raman probe as shown in Figure 1. As mentioned in the previous section, the hardware design of the sensor was based on the compatibility of the sensor to the ARAKNES robot. The specifications for the sensor were that (i) the length of the fiber probe should be approximately $5 \mathrm{~m}$ so that the laser and spectrometer could be kept within a safe distance from the operating table, (ii) the probe should be flexible, with a maximum length of $50 \mathrm{~mm}$ for the longest rigid section of the probe head, to ensure manoeuvrability of the probe by the robotic arm inside the abdominal cavity, and (iii) the diameter of the probe must be $8 \mathrm{~mm}$ or less, as the access port in the robot for inserting probe into the body has a diameter of $8 \mathrm{~mm}$.

Based on these requirements, a custom designed Raman probe was obtained from a commercial company (Emvision LLC). It contained one excitation fiber with $200 \mu \mathrm{m}$ diameter 
and 7 collection fibers each with a $200 \mu \mathrm{m}$ diameter. The rigid probe head was $50 \mathrm{~mm}$ long with a diameter $3.8 \mathrm{~mm}$. The probe head contained a low-pass filter at the excitation side and a long-pass filter at the collection side to reduce the background fluorescence from the optical fiber. The fiber pigtail was $5 \mathrm{~m}$ long and the rear end of the probe was terminated by two SMA connectors to connect the probe to the laser and spectrometer. A GRIN lens at the tip of the probe head set the working distance to approximately $1 \mathrm{~mm}$. The size of the excitation beam at the sample was $500 \mu \mathrm{m}$.

Typically, a power level of $100 \mathrm{~mW}$ was coupled into the Raman probe by a diode laser operating at $785 \mathrm{~nm}$ (PD-LD, BRAND AND MODEL, maximum power up to $500 \mathrm{~mW}$ ). The collection signal from the fiber was directed to a spectrometer (Shamrock SR-303i, Andor Technology) using an f-number matcher. This spectrometer employed a 400 lines $/ \mathrm{mm}$ grating, blazed at $850 \mathrm{~nm}$ and was equipped with a deep depletion, back-illuminated and thermoelectrically-cooled CCD camera (Newton, Andor Technology) for the detection of the Raman signal.

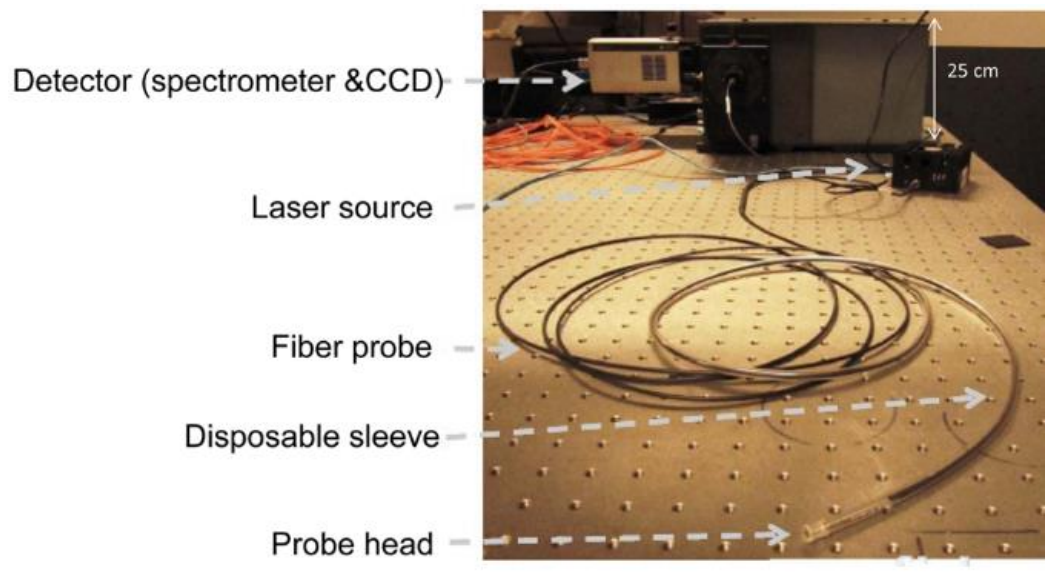

Figure 1: Hardware of the Raman bio-sensor.

\subsection{Disposable sleeve}

Maintaining the sterility of the probe is crucial for in vivo use during surgical intervention. The cost of this particular design of probe makes it not feasible to be used as a disposable probe and so a sterile disposable sleeve was provided into which the fiber probe could be inserted. The sterile sleeve was designed using a flexible tubing (Tygon, Cole-Parmer) having an inner diameter (ID) of $4.8 \mathrm{~mm}$ and outer diameter (OD) of $6.4 \mathrm{~mm}$. The rigid portion of the sleeve, constituting the probe head, was fabricated in poly (methyl methacrylate) (PMMA) with a 6.5 $\mathrm{mm}$ ID and a $7.5 \mathrm{~mm}$ OD and $10 \mathrm{~mm}$ length, to which the flexible tubing was attached. A sapphire window (Comar Optics, UK) of $7.5 \mathrm{~mm}$ diameter and $1 \mathrm{~mm}$ thickness was fixed to the other end of the rigid sleeve head. Both the flexible tubing and sapphire window were glued to the rigid sleeve tip using UV curable adhesive (Norland 60). The sapphire window provided a background-free optical access to the tissue when the fiber probe was inserted into the sleeve. Because the probe had a $1 \mathrm{~mm}$ working distance, with a $1 \mathrm{~mm}$ thick optical window, the probe+sleeve assembly couldbe operated as a contact probe brought into contact with the tissue when acquiring Raman spectral data. This provided a stable Raman signal amplitude by reducing the variability in the sample-probe distance. 
Another design consideration for the sleeve was the compatibility with a suitable surgical linsertion protocol. Indeed, during the insertion of the probe through the laparoscopic access port to the patient abdomen, the probe is not yet manoeuvred by the robot. It is therefore essential to keep the probe tip rigid, so that it can be driven within the field of view of the laparoscopic viewing system (camera) and within the grasping range of the arm of the surgical robot. Once the arm holds the tip of the probe, the probe should be sufficiently flexible to be maneuvered inside the abdomen cavity. To facilitate this, a guide wire system was introduced into the sleeve. PTFE microbore tubing (Cole Parmer) with $1.07 \mathrm{~mm}$ ID and $1.68 \mathrm{~mm}$ OD was inserted coaxially into the flexible sleeve tubing, acting as a self-lubricating channel for inserting the guide wire. Roslau piano wire of OD $1 \mathrm{~mm}$, annealed for increased elasticity, was used as the guide wire, which could be inserted and retracted into the PTFE microbore to control the sleeve rigidity. A schematic of the cross-section of the fiber sleeve is given in Figure 2a.

The total length of the sleeve was $1 \mathrm{~m}$, which was compatible with the requirements for the sterile area in the operating theatre and in the robot channels, and it was possible to insert the fiber probe directly into the sleeve without the need of any lubricant. Though this sleeve design is suitable for volume production as it is, it lends itself to high-volume production, where both fiber probe insertion channel and the guide wire insertion channel could be extruded as a single dual-lumen piece. A photograph of the sleeve with the fiber probe inserted is shown in Figure $2 b$.
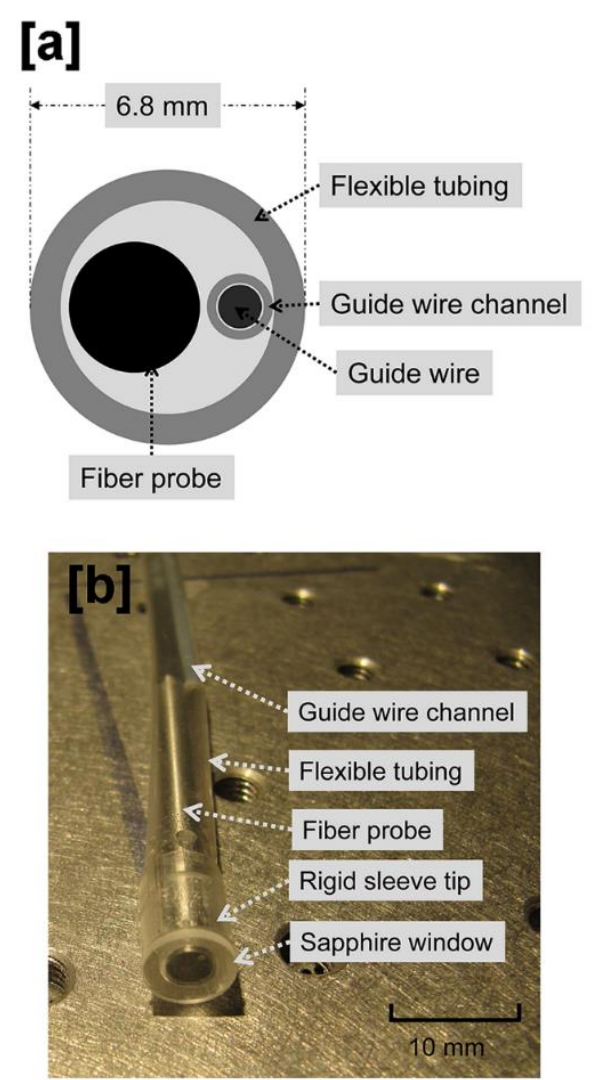

Figure 2: [a] Schematic of the disposable sleeve cross section. [b] Photograph of the disposable sleeve head into which the fiber probe was inserted 


\subsection{Mechanical compatibility to surgical robot}

The mechanical compatibility of the fiber probe with the sleeve was tested with the ARAKNES surgical robot. It was observed that the robotic arm could hold the Raman probe and that the probe was flexible enough to be manoeuvred by the robotic arm. The guide wire when inserted provided sufficient rigidity to the probe during insertion into the access port.

\section{Protocol and software}

A key objective was to develop a protocol that could be used by a surgeon who did not have detailed prior knowledge relating to Raman spectroscopy. Another consideration while developing the protocol was to ensure the highest possible sensitivity and specificity for this sensor for binary classification of tissues. One standard approach in tissue classification based on a supervised algorithm is to use a global training dataset to classify the query sample [7]. However, a downside with this approach is inter-patient variability in the bio-chemical composition of the tissue.

This sensor is designed to assist the surgeon in pin-pointing tissue margins. In particular,it assists the surgeon in identifying the separation line between two different tissue types, when such line location is otherwise visually ambiguous. This implies the clear presence of two tissue types (inside, type- $A$, and outside, type- $B$, the incision region) before the incision process takes place, and hence gives the opportunity to train the classifier using the two tissue regions from the patient itself. The protocol can therefore avoid inter-patient variability.

A flow chart of the three-step protocol is shown in Figure 3 . The creation of the training dataset involves positioning the probe at tissue type-A and recording Raman spectra, subsequntly recording Raman spectra from the type-B region. The Raman spectra acquired from the ambiguous region would then be classified using the training dataset.

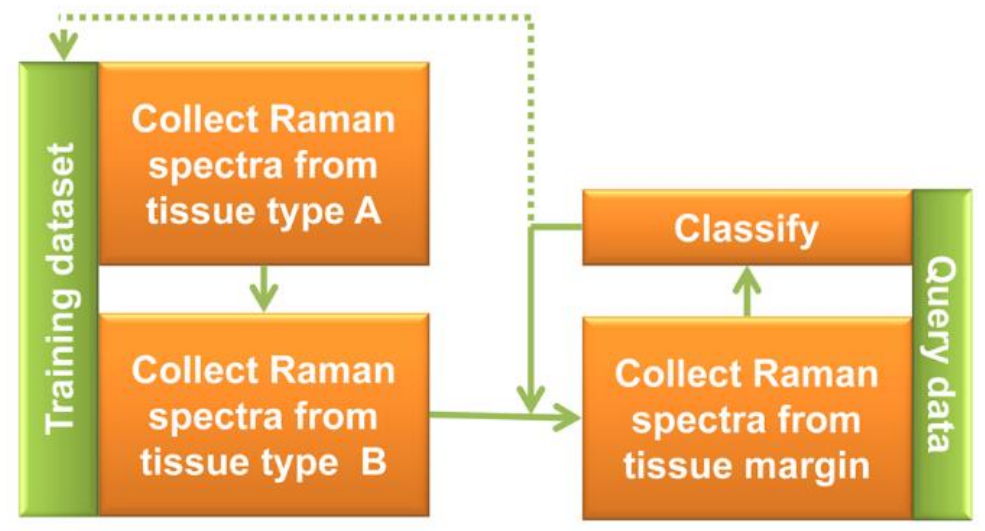

Figure 3: Flow chart of the protocol used in tissue classification

The training dataset includes a maximum of five Raman spectra each recorded from a single point on each of the two tissue types. Three Raman spectra would be acquired from each individual point while acquiring the query data and each of these three Raman spectra would be classified using the classification algorithm. 


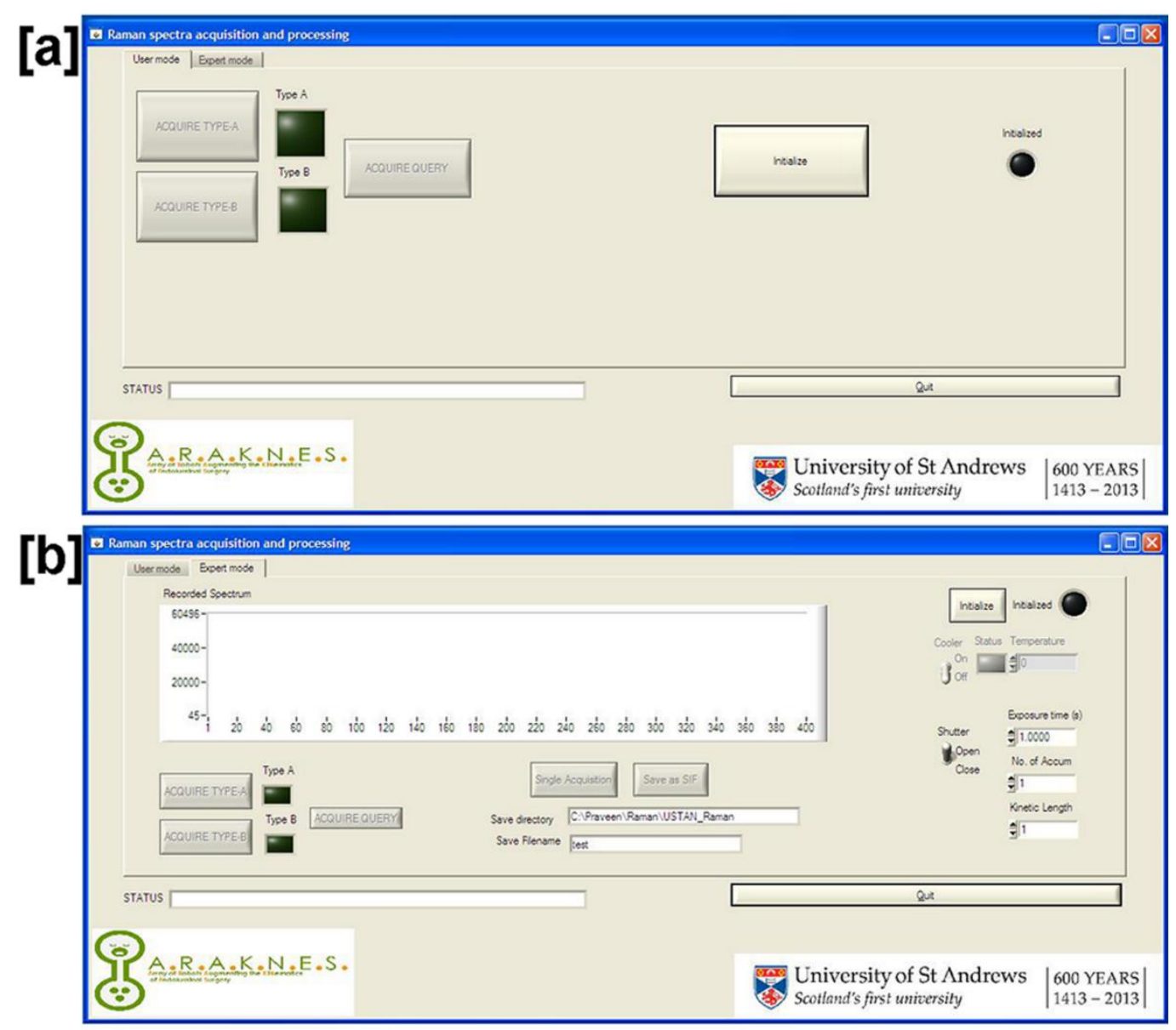

Figure 4: Screenshots of the tront-end intertace [a] User mode [b] Expert mode

Raman spectra within the region $500 \mathrm{~cm}^{-1}$ to $2000 \mathrm{~cm}^{-1}$ were used in testing the classification algorithm. All Raman spectra were post-processed by smoothing the data using a SavitzkyGolay filter and base-lining using an iterative modified polynomial fitting $[8,9]$.

In the classification algorithm, a Fisher Linear Discriminant Analysis (FLDA) was applied on the training dataset to reduce the dimensionality of the data. FLDA reduces the dimensionality in the feature space and transforms the multidimensional feature space into a one-dimensional feature space in such a way that the intra-class variance is minimized and the inter-class mean distance is maximized $[10,11]$. The transformation vector $\vec{v}$ obtained from the training dataset during this process was then applied to the query dataset as well to reduce to one dimension. Furthermore, the query data would be classified by finding the nearest mean distance to a class.

The software interface for this sensor was developed in Labwindows CVI 2009 (National Instruments). The front-end of the software interface can be run in two modes - either the User mode or the Expert mode. Screenshots of the front-end of the software interface are given in Figure 4. The Expert mode is designed to be used by an eventual service technician, where Raman acquisition parameters such as exposure time may be modified and the acquired Raman spectrum would be displayed. By comparison, the User mode had a minimalistic design, where there is no requirement to modify any settings such that once the system is initialized, a training dataset and query dataset may be acquired and the classified 
output be presented as a binary display. The user does not see the acquired Raman spectrum and does not require any expertise in analysing Raman spectroscopic data in the use of this system. A further operation mode, not described in this paper as not relevant to the present discussion, allows operation of an equivalent of the User mode through the robot operating console, thus rendering the full system transparent to the surgeon, who does not need to interact directly with the sensor acquisition system.

\section{Ex-vivo tissue analysis}

The efficacy of the Raman sensor to classify different tissue types was assessed at a proof-ofprinciple level by ex-vivo tissue analysis. The assessments were conducted in two modalities. Firstly, the Raman probe was positioned by hand over resected tissue samples and secondly, the Raman probe was mounted on a 6-axis robotic arm (VP-6242G Denso Robotics) and positioned on the tissue sample (Fig. 5).

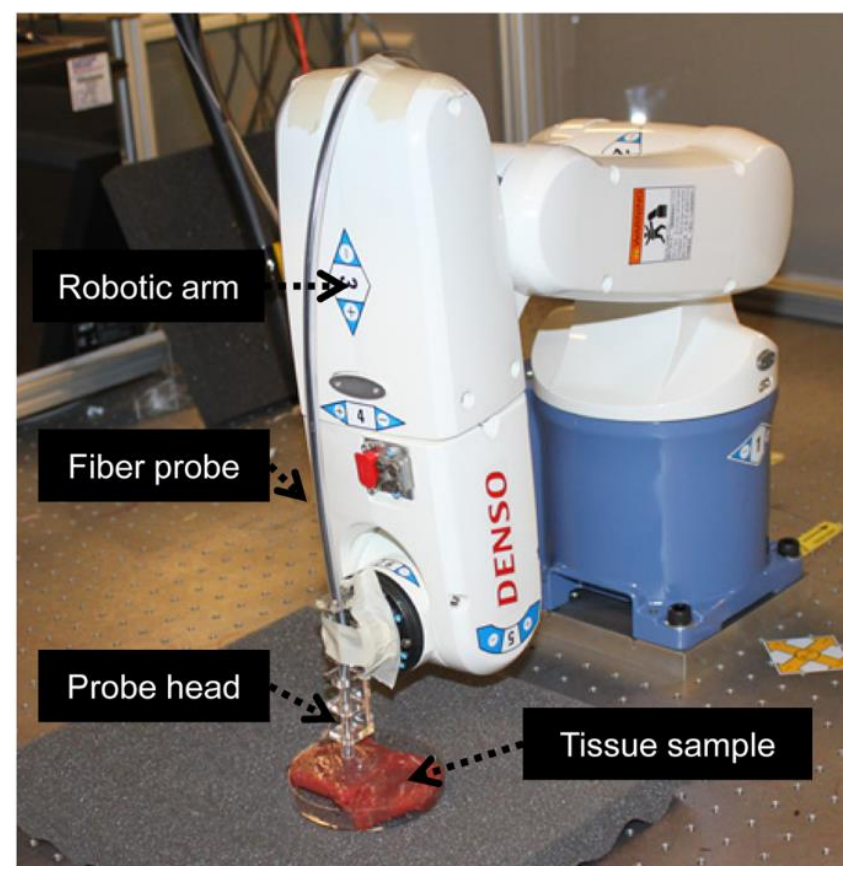

Figure 5: Photograph of the fiber probe mounted on the robotic arm to record Raman spectra from ex-vivo tissue samples

As per the protocol defined for this sensor, there would not be any pre-recorded training dataset. This makes it necessary to validate the algorithm for several training datasets. The ex-vivo tissue classification procedure was designed in the following manner. All the tissue samples used in these studies were sourced from a local butcher, certified for human consumption. The tissue samples had a thickness between $10-15 \mathrm{~mm}$ and were placed in $100 \mathrm{~mm}$ diameter petri dishes. One tissue type would be considered as type-A and the other tissue type would be considered as type-B. A training dataset was recorded from one point on each tissue type. Subsequently, ten query data points were recorded from each of the tissue types, in a randomized sequence in order to avoid any spurious correlation of the classifier. The acquired query data points were classified using the training dataset, and a 
confusion matrix was generated. Care was taken to ensure that none of the query data was acquired from same point in the tissue sample and none of the query data was recorded from the tissue region used for recording the training dataset. An exposure time of $1 \mathrm{~s}$ at a 100$\mathrm{mW}$ excitation power level at the sample was used for all of the measurements detailed in this section.

Table 1 shows the results of the assessment when the probe was positioned by hand on the tissue. It can be seen that the sensor gave a classification result having an accuracy exceeding $95 \%$ even for similar tissue samples such as bovine and lamb adipose tissues [12].

Because the sensor was developed to be manoeuvred by a robotic arm, a second assessment was carried out, where the probe was positioned using a robotic arm, as shown in Figure 5. This differs from a hand positioning, as the robot controls the absolute location of the probe, ratjer than the pressure and/or the relative location between probe and tissue, as a hand manipulation does. The protocol for testing the sensor remained same as the one described before and the results of the tissue classification are as given inTable 2, where it can be seen that the accuracy was above $95 \%$ in this case as well.

Table 1: Results of ex-vivo tissue classification for data acquired by positioning the probe by hand.

\begin{tabular}{|l|l|l|l|l|}
\hline $\begin{array}{l}\text { Positive } \\
\text { class }\end{array}$ & $\begin{array}{l}\text { Negative } \\
\text { class }\end{array}$ & Sensitivity & Specificity & Accuracy \\
\hline $\begin{array}{l}\text { Bovine } \\
\text { adipose }\end{array}$ & $\begin{array}{l}\text { Bovine } \\
\text { muscle }\end{array}$ & $100 \%$ & $100 \%$ & $100 \%$ \\
\hline $\begin{array}{l}\text { Porcine } \\
\text { adipose }\end{array}$ & $\begin{array}{l}\text { Porcine } \\
\text { muscle }\end{array}$ & $100 \%$ & $100 \%$ & $100 \%$ \\
\hline $\begin{array}{l}\text { Porcine } \\
\text { adipose }\end{array}$ & $\begin{array}{l}\text { Porcine } \\
\text { skin }\end{array}$ & $100 \%$ & $100 \%$ & $100 \%$ \\
\hline $\begin{array}{l}\text { Porcine } \\
\text { adipose }\end{array}$ & $\begin{array}{l}\text { Bovine } \\
\text { adipose }\end{array}$ & $100 \%$ & $100 \%$ & $100 \%$ \\
\hline $\begin{array}{l}\text { Bovine } \\
\text { adipose }\end{array}$ & $\begin{array}{l}\text { Lamb } \\
\text { adipose }\end{array}$ & $96 \% \pm 6 \%$ & $95 \% \pm 5 \%$ & $\begin{array}{l}95 \% \\
5 \%\end{array}$ \\
\hline $\begin{array}{l}\text { Bovine } \\
\text { muscle }\end{array}$ & $\begin{array}{l}\text { Lamb } \\
\text { muscle }\end{array}$ & $95 \% \pm 5 \%$ & $90 \% \pm 7 \%$ & $85 \%$ \\
\hline
\end{tabular}


Table 2: Results of ex-vivo tissue classification for data acquired by positioning probe using a robotic arm.

\begin{tabular}{|l|l|l|l|l|}
\hline $\begin{array}{l}\text { Positive } \\
\text { class }\end{array}$ & $\begin{array}{l}\text { Negative } \\
\text { class }\end{array}$ & Sensitivity & Specificity & Accuracy \\
\hline $\begin{array}{l}\text { Bovine } \\
\text { adipose }\end{array}$ & $\begin{array}{l}\text { Bovine } \\
\text { muscle }\end{array}$ & $100 \%$ & $100 \%$ & $100 \%$ \\
\hline $\begin{array}{l}\text { Porcine } \\
\text { adipose }\end{array}$ & $\begin{array}{l}\text { Porcine } \\
\text { muscle }\end{array}$ & $100 \%$ & $100 \%$ & $100 \%$ \\
\hline $\begin{array}{l}\text { Porcine } \\
\text { adipose }\end{array}$ & $\begin{array}{l}\text { Bovine } \\
\text { adipose }\end{array}$ & $\begin{array}{l}95 \% \pm \\
10 \%\end{array}$ & $\begin{array}{l}95 \% \pm \\
10 \%\end{array}$ & $\begin{array}{l}95 \% \pm \\
10 \%\end{array}$ \\
\hline
\end{tabular}

\section{Discussions}

It can be seen from the mechanical tests and ex-vivo tissue classification tests that the Raman bio-sensor developed for ARAKNES is effective and the protocol developed implied that a user with no prior spectroscopy experience would be able to use it. One of the key advantages of this protocol is that it is not limited to a specific tissue type. Given that the training dataset is acquired locally, this sensor may be used to distinguish between any two tissue types provided there is variation in the bio-chemical compositions.

A wide range of literature over last decade has confirmed that Raman spectroscopy can be used in the discrimination of normal and cancerous tissues for a variety of tissue sites through in-vivo and ex-vivo studies [13-16]. In principle, the Raman bio-sensor developed in this collaboration could be used for all of these applications. The sensor design is modular and so this facilitates independent upgrades of the hardware (fiber Raman probe, laser or spectrometer) or software (classification algorithm) if the sensitivity of the system has to be enhanced for specific applications.

\section{Conclusion}

Photonics-based sensing tools have the potential to provide a wealth of complementary information regarding the mechanical and chemical properties of tissues that would assist surgeons in making better informed decisions during surgical procedures. Here, we have demonstrated the development of a Raman-based bio-sensor that offers a means for tissue discrimination in endoluminal robotic surgery where the hardware of this system has been designed to be compatible with ARAKNES surgical robot. A disposable sleeve was designed to ensure the sterility of the probe. Given that the end-user of this sensor would be a surgeon (possibly without detailed experience of spectroscopy), we defined a protocol that gives binary information. The software interface developed had an 'expert' and 'user' mode, where the user mode could be used in a straightforward way without going into the complexities of Raman spectroscopic techniques. The protocol creates a training dataset locally from the tissue from the same patient for the supervised classifier. This avoids inter-patient variability of Raman information and makes the sensor generic for use in binary discrimination between any types of tissues. The system was tested to be mechanically compatible with the ARAKNES 
robot and a better than 95\% accuracy was demonstrated in a proof-of-principle ex-vivo tissue classification assessment, both when the probe is held by hand and when it is manuipulated by a robotic arm. This sensor thus represents a generic platform for the exploitation of Raman spectroscopy as a complementary technique for tissue discrimination in laparoscopic surgery.

\section{Acknowledgements}

We acknowledge EU funding under grant agreement nr. 224565 "ARAKNES - Array of Robots Augmenting the KiNematics of Endoluminal Surgery",. We would like to thank Dr. Gajendra Pratap Singh and Dr. Tom Brown for their involvement in the initial part of this study. We would also like to thank Mr. Gianluigi Petroni for testing the mechanical compatibility of the probe with the ARAKNES robot.

\section{References}

[1] C. Krafft, and V. Sergo, Spectrosc-Int J 20, 195 (2006).

[2] J. T. Motz, M. Hunter, L. H. Galindo, J. A. Gardecki, J. R. Kramer, R. R. Dasari, and M. S. Feld, App. Optics 43, 542 (2004).

[3] J. T. Motz, S. J. Gandhi, O. R. Scepanovic, A. S. Haka, J. R. Kramer, R. R. Dasari, and M. S. Feld, J. Biomed. Opt. 10 (2005).

[4] A. Mahadevan-Jansen, M. F. Mitchell, N. Ramanujam, U. Utzinger, and R. RichardsKortum, Photochem. Photobiol. 68, 427 (1998).

[5] L. A. Reisner, B. W. King, M. D. Klein, G. W. Auner, and A. K. Pandya, Int J Med Robot Comp 3, 82 (2007).

[6] www.araknes.org, last visited on 22 January 2013.

[7] N. Stone, C. Kendall, J. Smith, P. Crow, and H. Barr, Faraday Discussions 126, 141 (2004).

[8] C. A. Lieber, and A. Mahadevan-Jansen, Appl. Spectrosc. 57, 1363 (2003).

[9] P. C. Ashok, G. P. Singh, H. A. Rendall, T. F. Krauss, and K. Dholakia, Lab Chip 11, 1262 (2011).

[10]J. Liefeng BoLing WangLicheng, Neural Computation 18, 961 (2006).

[11]S. Mika, G. Ratsch, J. Weston, B. Scholkopf, and K. R. Mullers, Neural Networks for Signal Processing IX, 1999. Proceedings of the 1999 IEEE Signal Processing Society Workshop, 41 (1999).

[12]J. R. Beattie, S. E. J. Bell, C. Borggaard, A. M. Fearon, and B. W. Moss, Lipids 42, 679 (2007). [13] (!!! INVALID CITATION !!!).

[14]M. S. Bergholt, W. Zheng, K. Lin, K. Y. Ho, M. Teh, K. G. Yeoh, J. B. Y. So, and Z. W. Huang, J. Biomed. Opt. 16 (2011).

[15]H. L. a. D. I. M. Haishan Zeng, (SPIE, 2011).

[16]R. O. P. Draga, M. C. M. Grimbergen, P. L. M. Vijverberg, C. F. P. v. Swol, T. G. N. Jonges, J. A. Kummer, and J. L. H. Ruud Bosch, Anal. Chem. 82, 5993 (2010). 